\title{
KNOWLEDGE AND REASONING IN PROGRAM SYNTHESIS
}

BY

ZOHAR MANNA, Applied Mathematics Department, Weizmann Institute of Science, Rehovot, Israel

and

RICHARD WALDINGER, Artificial Intelligence Center, Stanford Research Institute, Menlo Park, California, U. S. A.

\section{ABSTRACT:}

Program synthesis is the construction of a computer program from given specifications. An automatic program synthesis system must combine reasoning and programning ability with a good deal of knowledge about the subject matter of the program. This ability and knowledge must be represented both procedurally (by programs) and structurally (by choice of representation).

We describe some of the reasoning and programming capabilities of a projected synthesis system. Special attention is paid to the introduction of conditional tests, loops, and intructions with side effects in the program being constructed. The ability to satisfy several interacting goals simultaneously proves to be important in many contexts. The modification of an already existing program to solve a somewhat different problem has been found to be a powerful approach.

We illustrate these concepts with hand simulations of the synthesis of a number of pattem-matching programs. Some of these techniques have already been implemented, others are in the course of implementation, while others seem equivalent to wellknown unsolved problems in artificial intelligence. 


\section{INTRODUCTION}

In this paper we describe some of the knowledge and the reasoning ability that a computer system must have in order to construct computer programs automatically. It is our hypothesis that such a system needs to embody a relatively small class of reasoning and programming tactics combined with a great deal of knowledge about the world. These tactics and this knowledge are expressed both procedurally (i.e., explicitly in the description of a problem. solving process) and structurally (i.e., implicitly in the choice of representation). We consider the ability to reason as central to the program synthesis process, and nost of this paper is concerned with the incorporation of common-sense reasoning techniques into a program synthesis system. However, symbolic reasoning alone will not suffice to produce the synthesis of complex programs; we therefore consider other techniques as we11:

- The construction of "almost correct" programs that must be debugged (cf. Sussman [1973]).

- The modification of an existing program to perform a somewhat different task (cf. Balzer [1972]).

- The use of "visual" representations to reduce the need for deduction (cf. Bundy [1973]).

We regard program synthesis as a part of antificial intelligence. Many of the abilities we require of a program synthesizer, such as the ability to represent knowledge or to draw common-sense conclusions from facts, we would also expect from a natural language understanding system or a robot problem solver. These general problems have been under study by researchers for many years, and we do not expect that they will all be solved in the near future. However, we still prefer to address those problems rather than restrict ounselves to a more limited program synthesis system without those abilities.

Thus, although implementation of some of the techniques in this paper has aiready been completed, others require further development before a complete implementation will be possible. We imagine the knowledge and reasoning tactics of the system to be expressed in a PLANNER-type language (Hewitt [1972]); our own 
implementation is in the QLISP language (Reboh and Sacerdoti [1973]). Further details on the implementation are discussed in Section $\mathrm{V}-\mathrm{A}$.

Part II of the paper gives the basic techniques of reasoning for program synthesis. They include the formation of conditional tests and loops, the satisfaction of several simultaneous goals, and the handing of instructions with side effects. Part III applies the techniques of Part II to synthesize a nontrivial "pattern-matcher" that determines if a given expression is an instance of a given pattern. We show how different choices made during the synthesis process result in different final programs. Part IV demonstrates the modification of programs. We take the pattern matcher we have constructed in Part III and adapt it to construct a more complex program: a "unification algorithm" that determines if two patterns have a common instance. In Part $V$ we give some of the historical background of automatic program synthesis, and we compare this work with other recent efforts.

\section{FUNDAMENTAL REASONING}

In this section we will describe some of the reasoning and programming tactics that are basic to the operation of our proposed synthesizer. These tactics are not specific to one particular domain; they apply to any programing problem. In this class of tactics, we include the formation of program branches and loops and the handing of statements with side effects.

\section{A. Specification and Tactics Language}

We must first say something about how programing problems are to be specified. In this discussion we consider only correct and exact specifications in an antificial language. Thus, we will not discuss input-output examples (cf. Green et al. [1974], Hardy [1974]), traces (cf. Biermann et al. [1973]), on natural language descriptions as methods for specifying programs; nor will we consider interactive specification of programs (cf. Balzer [1972]). Neither are we limiting ourselves to the first-order predicate calculus (of. Kowalski [1974]). Instead, we try to introduce specification constructs that allow the natural and 
intuitive description of programing problems. We therefore include constmucts such as

Find $x$ such that $P(x)$

and the ellipsis notation, e.g.,

$$
\mathrm{A}[1], \mathrm{A}[2], \ldots, \mathrm{A}[\mathrm{n}] \text {. }
$$

Furthermore, we introduce new constructs that are specific to certain subject domains. For instance, in the domain of sets we use

$$
\{x \mid P(x)\}
$$

for "the set of all $x$ such that $P(x)$ ". As we introduce an example we will describe features of the language that apply to that example. Since the specification language is extendible, we can introduce new constructs at any time.

We use a separate language to express the system's knowledge and reasoning tactics. In the paper, these will be expressed in the form of rules written in English. In our implementation, the same rules are represented as programs in the QLISP programming language. When a problem or goal is presented to the system, the appropriate rules are summoned by "pattern-directed function invocation" (Hewitt [1972]). In other wonds, the form of the goal determines which rules are applied.

In the following two sections we will use a single example, the synthesis of the set-theoretic union program, to illustrate the formation both of conditionals and of loops. The problem here is to compute the union of two finite sets, where sets are represented as lists with no repeated elements.

Given two sets, $s$ and $t$, we want to express union $(s t)=\{x \mid x \in s$ or $x \in t\}$

in a LISP-like language. We expect the output of the synthesized program to be a set itself. Thus

$$
\text { union }((A B) \quad(B C))=(A B C) \text {. }
$$

We do not regard the expression $\{x \mid x \in s$ or $x \in t\}$ itself as a proper program: the operator $\{\mid \ldots\}$ is a construct in our specification language but not in our LISP-like programming language. We assume that the programming language does have the following functions: 
head $(2)=$ the first element of the list $l$.

Thus head $((A B C D))=A$.

tail $(Q)=$ the list of all but the first element of the list $l$.

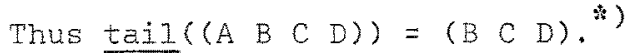

$\operatorname{add}(x, s)=$ the set consisting of the element $x$ and the elements of the set $s$.

Thus $\operatorname{add}(A(B) C D))=\left(\begin{array}{llll}A & B & C & D\end{array}\right)$

whereas $\operatorname{add}(B(B \quad C \quad D))=\left(\begin{array}{lll}B & C & D\end{array}\right)$.

empty(s) is true if $s$ is the empty list

false otherwise.

Our task is to transform our specifications into an equivalent algorithm in this programming language.

We assume the system has some basic knowledge about sets, such as the following rules:

(1) $x \in S$ is false if empty (s)

(2) $x \in s$ is equivalent to $(x=$ head(s) or $x \varepsilon$ tail (s)) if $\sim \operatorname{empty}(s)$.

(3) $\{x \mid x \in s\}$ is equal to $s$

(4) $\{x \mid x=$ a or $Q(x)\}$ is equal to add $(a\{x \mid Q(x)\})$

We also assume that the system knows a considerable amount of propositional logic, which we will not mention explicitly.

Before proceeding with our example we must discuss the formation of conditional expressions.

\section{B. Formation of Conditional Expressions}

In addition to the above constructs, we assume that our programming language contains conditional expressions of the form

$$
\text { (if } p \text { then } q \text { else } r \text { ) }=r \text { if } p \text { is false }
$$

q otherwise.

The conditional expression is a technique for dealing with uncertainty. In constructing a program, we want to know if condition $p$ is true or not, but in fact $p$ may be true on some occasions

Wince sets are represented as lists, head and tail may be applied to sets as well as lists. Their value then depends on our actual choice of representation. 
and false on others, depending on the value of the argument. The human programmer faced with this problem is likely to resort to "hypothetical reasoning": he will assume $p$ is false and write a program $r$ that solves his problem in that case; then he will assume $p$ is true and write a program $q$ that works in that case; he will then put the two programs together into a single program

\section{(if $p$ then $q$ else $r$ ).}

Conceptually he has solved his problem by splitting his world into two worlds: the case in which $p$ is true and the case in which $\mathrm{p}$ is false. In each of these worlds, uncertainty is reduced. Note that we must be careful that the condition $\mathrm{p}$ on which we are splitting the world is computable in our programing language; otherwise, the conditional expression we construct also will not be computable (cf. Luckham and Buchanan [1974]).

We can now proceed with the synthesis of the union function. Our specifications were

$$
\text { union }(s t)=\{x \mid x \in s \text { or } x \varepsilon t\} \text {. }
$$

We begin to transform these specifications into an equivalent program in our language, using our xules. We examine the subexpression $\mathrm{x} \varepsilon \mathrm{s}$. Two of the rules, (1) and (2), apply to this subexpression. Rule (1) generates a subgoal, empty(s). We cannot prove $s$ is empty - this depends on the input -- and therefore this is an occasion for a hypothetical world split. (We know that empty(s) is a computable condition becuase empty is a primitive in our language.) In the case in which $s$ is empty, the expression

$$
\{x \mid x \in s \text { or } x \in t\}
$$

therefore reduces to

$$
\{x \mid \text { false or } x \in t\} \text {, }
$$

or, by propositional logic,

$$
\{x \mid x \in t\} \text {. }
$$

Now rule (3) reduces this to $t$, which is one of the inputs to our program and therefore is itself an acceptable program segment in our language.

In the other world--the case in which $s$ is not empty--we cannot solve the problem without discussing the recursive loop formation 
mechanism. However, we know at this point that the program will have the form

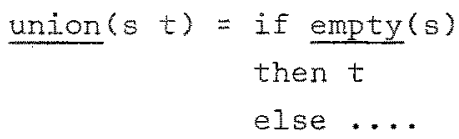

where the else clause will be whatever program segment we construct for the case in which $s$ is not empty.

Before we continue with this example we will discuss the loop formation mechanism.

\section{c. Formation of LoOpS}

The term "loop" includes both iteration and recursion; however, in this paper we will only discuss recursive loops (cf. Manna and Waldinger [1971]). Intuitively, we form a recursive call when, in the course of working on our problem, we generate a subgoal that is identical in form to our top-level goal. For instance, suppose our top-level goal is to construct the program reverse $(l)$, that reverses the elements of the list \& $(e . g \cdot$, reverse $(A(B) C) D)=$ $(D(B C) A))$. If in the course of constructing this program we generate the subgoal of reversing the elements of the list tail( $(l)$, we can use the program we are constructing to satisfy this subgoal. In other words we can introduce a recursive call reverse (tail(l)) to solve the subsidiary problem. We must always check that a recursive call cannot lead to an infinite recursion. No such infinite loop can occur here because the input tail(l) is "shorter" than the original input $\ell$.

Let us see how this technique applies to our union example. Continuing where we left off in the discussion of conditionals, we attempt to expand the expression

$$
\{x \mid x \in s \text { or } x \in t\}
$$

in the case in which $s$ is not empty. Applying rule (2) to the subexpression $x \in s$, we can expand our expression to

$$
\{x \mid x=\operatorname{head}(s) \text { or } x \in \operatorname{tail}(s) \text { or } x \varepsilon t\} \text {. }
$$

Using rule (4), this reduces to

If we observe that

$$
\text { add (head(s) }\{x \mid x \in \text { tail (s) or } x \in t\} \text { ). }
$$

$$
\{x \mid x \in \operatorname{tai}(s) \text { or } x \in t\}
$$


is an instance of the top-level subgoal, we can reduce it to union (tail (s) t).

Again, this recursive call leads to no infinite loops, since tail(s) is shorter than $s$. Our completed union program is now

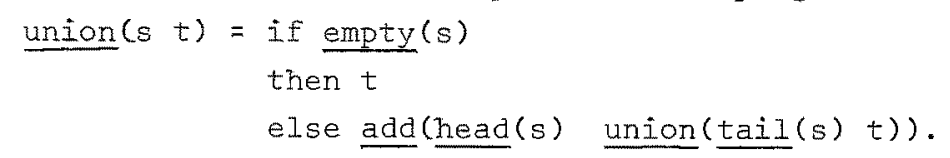

As presented in this section, the loop formation technique can only be applied if a subgoal is generated that is a special case of the top-level goal. We shall see in the next section how this restriction can be relaxed.

\section{Generalization of Specifications}

When proving a theorem by mathematical induction, it is often necessary to strengthen the theorem in order for the induction to "go through." Even though we have an apparently more difficult theorem to prove, the proof is facilitated because we have a stronger induction hypothesis. For example, in proving theorems about LISP programs, the theorem prover of Boyer and Moore [1973] often automatically generalizes the statement of the theorem in the course of a proof by induction.

A similar phenomenon occurs in the synthesis of a recursive program. It is often necessary to strengthen the specifications of a program in order for that program to be useful in recursive calls. We believe that this ability to strengthen specifications is an essential part of the synthesis process, as many of our examples will show.

For example, suppose we want to construct a program to reverse a list. A good recursive reverse program is

where

$$
\text { reverse }(l)=\text { rev }(l())
$$

$$
\begin{aligned}
\underline{\operatorname{rev}}(l \mathrm{~m})= & \text { if } \frac{\operatorname{empty}}{\text { then } \mathrm{m}}(l) \\
& \text { else } \operatorname{rev}(\operatorname{tail}(l) \operatorname{head}(l) \cdot \mathrm{m}) .
\end{aligned}
$$

Here 
() is the empty list

$x \cdot \ell$ is the list formed by inserting $x$ before the first element of $2 .(e . g ., A \cdot(B C D)=(A B C D))$.

Note that mev $(l \mathrm{~m})$ reverses the 1 ist $l$ and appends it onto the list $\mathrm{m}, \mathrm{e} \cdot \mathrm{g} . \mathrm{s}$

$$
\operatorname{rev}((A B C)(D E))=(C B A D E)
$$

This is a good way to compute reverse: it uses very primitive LISP functions and its recursion is such that it can be compiled without use of a stack. However, writing such a program entails writing the function rev, which is apparently more general and difficult to compute than reverse itself, since it must reverse its first argument as a subtask. The synthesis of this reverse function involves generalizing the original specifications of reverse into the specifications of rev.

The reverse function requires that the top-level goal be generalized in onder to match the lower level goal. Another way for the specifications to be generalized is as follows. Suppose in the course of the synthesis of a function $f(x)$, we generate a subgoal of the form $P(f(a))$, where $f(a)$ is a particular recursive call. Instead of proving $P(f(a))$, it may be easien to rewrite the specifications for $f(x)$ so as to satisfy $P(f(x)$ ) for all $x$. This step may require that we actually modify portions of the program that have already been synthesized in order to satisfy the new specification $P$. The recursive call to the modified program will then be sure to satisfy $P(f(a))$. This process will be illustrated in more detail during the synthesis of the pattem matcher in Part III.

\section{E. Conjunctive Goals}

The problem of solving conjunctive goals is the problem of synthesizing a program that satisfies several constraints simultaneously. The general form for this problem is

Find $z$ such that $P(z)$ and $Q(z)$.

The conjunctive goals problem is difficult because, even if we have methods for solving the goals

Find $z$ such that $P(z)$

and 
Find $z$ such that $Q(z)$

independently, the two solutions may not merge together nicely into a single solution. Moreover, there seems to be no way of solving the conjunctive goal problem in general; a method that works on one such problem may be irrelevant to another.

We will illustrate one instance of the conjunctive goals problem: the solution of two simultaneous linear equations. Although this problem is not itself a program synthesis problem, it could be rephrased as a synthesis problem. Moreover the difficulties involved and the technique to be applied extend also to many real synthesis problems, such as the pattern-matcher synthesis of Part III. Suppose our problem is the following:

$$
\text { Find } \begin{aligned}
& \left\langle z_{1}, z_{2}\right\rangle \text { such that } \\
& 2 z_{1}=z_{2}+1 \text { and } \\
& 2 z_{2}=z_{1}+2 .
\end{aligned}
$$

Suppose further that although we can solve single linear equations with ease, we have no built-in package for solving sets of equations simultaneously. We may try first to find a solution to each equation separately. Solving the first equation, we rnight come up with

$$
\left\langle z_{1}, z_{2}\right\rangle=\langle 1, I\rangle \text {, }
$$

whereas solving the second equation might give

$$
\left\langle z_{1}, z_{2}\right\rangle=\langle 2,2\rangle \text {. }
$$

There is no way of combining these two solutions. Furthermore, it doesn't help matters to reverse the order in which we approach the two subgoals. What is necessary is to make the solution of the first goal as general as possible, so that some special case of the solution might satisfy the second goal as well. For instance, a "general" solution to the first equation might be

$$
\langle I+w, I+2 w>\text { for any } w \text {. }
$$

This solution is a generalization of our earlier solution $\langle 1,1\rangle$. The problem is how to find a special case of the general solution that also solves the second equation. In other words, we must find a w such that

$$
2(1+2 w)=(1+w)+2 \text {. }
$$

This strategy leads us to a solution.

Of course the method of generalization does not apply to all conjunctive goal problems. For instance, the synthesis of an inte- 
ger square-root program has specifications

Find $z$ such that

$z$ is an integer and

$z^{2} \leqslant x$ and

$(z+1)^{2}>x$

where $x \geqslant 0$.

The natural approach of finding a general solution to one of the conjuncts and plugging it into the others is not practical in this case.

\section{F. Side Effects}

Up to now we have been considering programs in a LISP-Iike language. These prognams return a value but have no side effects. In the next two sections we will consider the synthesis of more general programs which may modify the state of the world. Programs that change the values of variables or alter the configuration of data structures are examples of this class. This sort of program is usually synthesized when a goal is proposed of the general form

\section{Achieve P.}

A program that satisfies this specification will have the effect of making $P$ true.

To discuss this general case we will continue to use the concept of "world" that we introduced in our discussion of hypothetical reasoning. The concept of world is virtually identical to the concept of state (McCarthy [1962]). Assertions may be true in one world and false in another. New worlds may be constructed by programs in three ways: world modification, splitting and joining.

- World Modification - - The execution of an instruction with side effects causes the creation of a new world. None of the assertions in the old world may be assumed to be true in the new world.

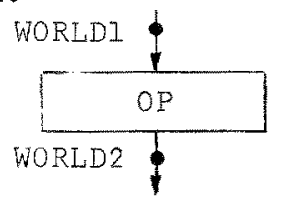


- World Splitting -- The execution of a conditional test $P$ causes the creation of two new worlds.

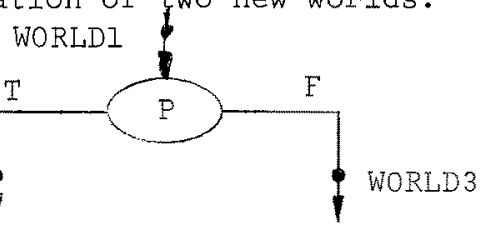

Any assertion in WORLD 1 is also true in WORLD2 and WORLD3. Furthermore $\mathrm{P}$ is true in wORLD2 and $\sim \mathrm{P}$ is true in WORLD3.

- World Joining -- When two paths of a program join together, the corresponsing worlds are joined too.

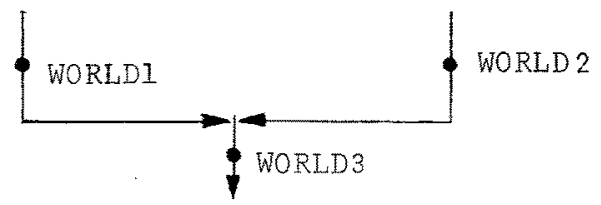

Here, in order for an assertion to be true in WORLD3 it must be true in both WORLD1 and WORLD2.

When an instruction with side effects has modified the world, we need to be able to test if an assertion is true in the new world after the instruction is executed. We do this by "passing" that assertion back to the old world before the instruction is executed. When we "pass" an assertion back over an instruction, we actually construct a new assertion that must be true in the old world in order that the oniginal assertion will be true in the new world (cf. Floyd [1967] and Hoare [1969]).

To illustrate the application of these constructs to the synthesis of a program with side effects, let us consider the program sort $(x y)$ that sorts the values of two variables $x$ and $y$. For simplicity we will use the statement interchange $(x y)$ that exchanges the value of $x$ and $y$, instead of primitive assignment statements. Our specifications will be simply

Achieve $x \leqslant y$.

Strictly speaking, we should include in the specifications the additional requirement that the set of values of $x$ and $y$ after the sort should be the same as before the sort. However, we will not consider such complex goals until the next section, and we can achieve the same effect by requiring that the interchange statement be the only instruction with side effects that appears in the program. 
The first step in achieving a goal is to see if it is already true. (If a goal is a theorem, for instance, we do not need to construct a program to achieve it.) We cannot prove $x \leqslant y$, but we can use it as a basis for a hypothetical world split. Our program thus far is

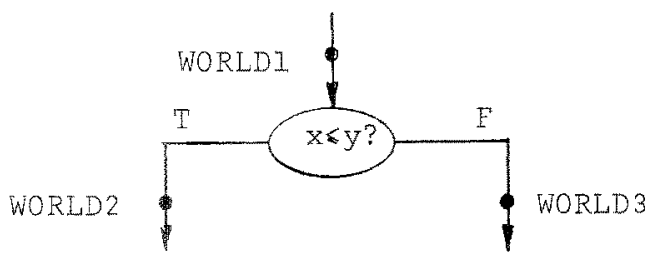

In WORLD2 our goal is already achieved; we may restrict our attention to WORLD3. In WORLD3 we know that (x<y), i.e., $x>y$. To achieve $x \leqslant y$, it suffices to establish $x<y$, but this may be achieved by executing interchange $(x, y)$, creating WORLD4 .

WORLDI

WORLD2

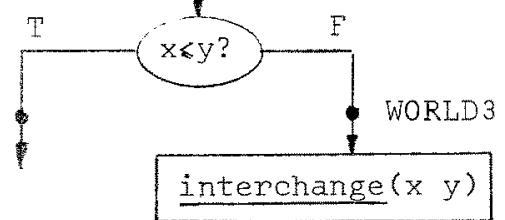

WORLD4

We have achieved $x<y$ in both WORLD2 and WORLD4. If we join them together, we will have succeeded in modifying wORLDI to make $x \leqslant y$ true. The final program is therefore:

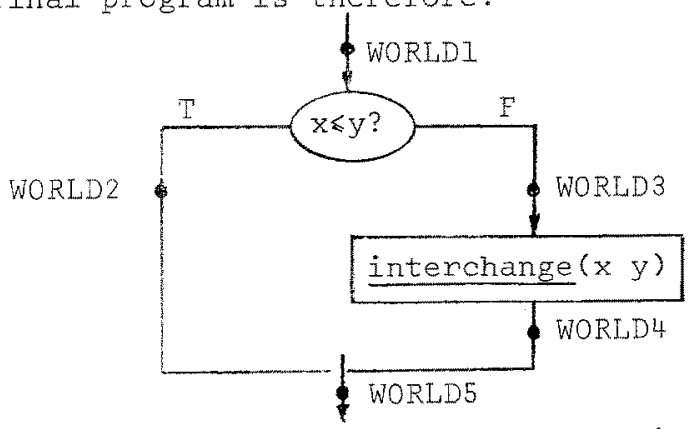

Often a goal to be achieved will involve the simultaneous satisfaction of more than one condition. As in the case of the conjunctive goals in the programs without side effects, the special 
interest of this problem lies in the interaction between the subgoals. The satisfaction of simultaneous goals will be the subject of the next section.

\section{G. Simultaneous Goals}

The problem of simultaneous goals is the problem of approaching a goal of form

Achieve $P$ and $Q$.

Sometimes $P$ and $Q$ will be independent conditions, so that we can achieve $P$ and $Q$ simply by achieving $P$ and then achieving $Q$. For example, if our goal is

Achieve $\mathrm{x}=2$ and $\mathrm{y}=3$,

the two goals $x=2$ and $y=3$ are completely independent. In this section, however, we will be concerned with the more complex case in which $P$ and $Q$ interact. In such a case we may make $P$ false in the course of achieving $Q$.

Consider for example the problem of sorting three variables $x, y$, and $z$. We will assume that the only instruction we can use is the subroutine sont ( $u v$ ), described in the previous section, which sonts two variables. Our goal is then

Achieve $\mathrm{x} \leqslant \mathrm{y}$ and $\mathrm{y} \leqslant \mathrm{z}$.

We know that the program sort ( $u v$ ) will achieve a goal of form $u \leqslant v$. If we apply the straightforward technique of achieving the conjunct $x \leqslant y$ first, and then the conjunct $y \leqslant z$, we obtain the program

$$
\begin{aligned}
& \operatorname{sont}(x y) \\
& \text { sont }\left(\begin{array}{ll}
y & z
\end{array}\right) .
\end{aligned}
$$

However, this program has a bug in that sorting $y$ and $z$ may disrupt the relation $x<y$ : if $z$ is initially the smallest of the three, we make $y$ less than $x$ in interchanging $y$ and $z$. Reversing the onder in which the conjuncts are achieved is useless in this case. There are a number of ways in which this problem may be resolved. one of them involves the notion of debugging (cf. Sussman [1973]). The approach is to debug the program

$$
\begin{aligned}
& \operatorname{sont}(x y) \\
& \operatorname{sont}\left(\begin{array}{ll}
y & z
\end{array}\right)
\end{aligned}
$$

so that in executing sont $\left(\begin{array}{ll}y & z\end{array}\right)$ we do not disturb the relation 
$x \& y$. To illustrate the process more clearly, we will expand the definition of sort in terms of interchange and present the entire program in flowchart notation.

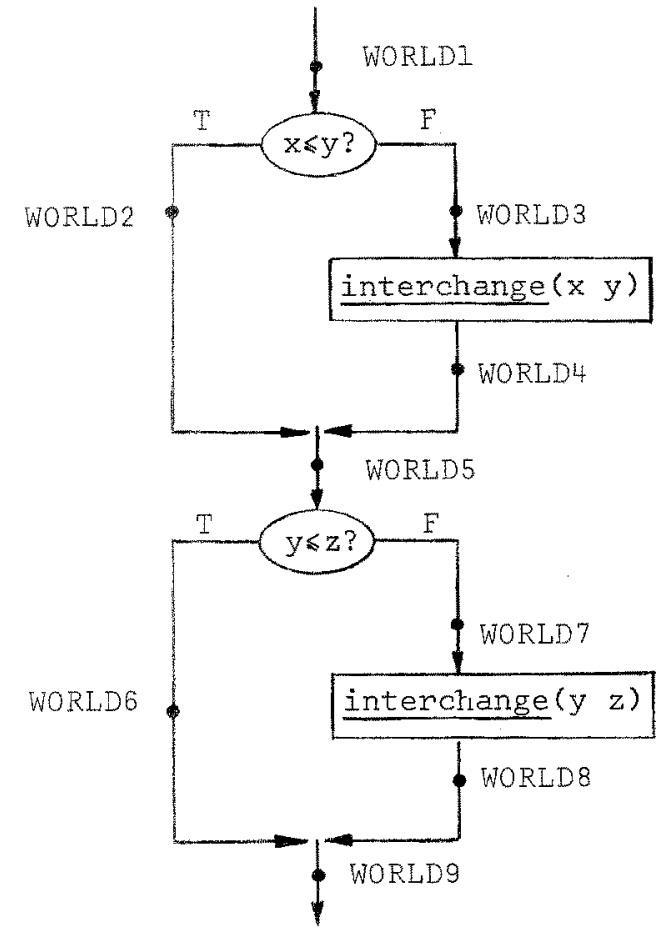

We want to modify this program so that $x$ y in wORLD9. We now choose not to achieve $x \leqslant y$ directly in WORLD9, for fean of disturbing the protected relation $y \leqslant z$. Instead we decide to pass the predicate $x \leqslant y$ back to some earlier point in the program where there are no protected relations. We can then safely attempt to achieve the modified predicate at that point.

There are two ways of passing the predicate $x<y$ back to WORLD5 -through WoRLD6 on through WORLD8 and WORLD7. In the first case the predicate is unmodified. Since we know $x<y$ is true in WORLD5, we do not have to worry about this case. In the second case, passing $x \leqslant y$ over statement interchange $(y z)$ gives the modified predicate $x \leqslant z$ in WORLD7. Therefore we must achieve $x \leqslant z$ if $y>z$ in WORLD5.

We may attempt to achieve this relation directly, by inserting the instruction sont $(x z)$ in the midale of the program, yielding 
the program

$$
\begin{aligned}
& \operatorname{sont}(x y) \\
& \operatorname{sort}(x z) \\
& \operatorname{sont}(y z) .
\end{aligned}
$$

However, this action, though ultimately correct, risks disturbing the relation $x \leqslant y$ protected in WORLD5. A more prudent course is to pass the predicate back still further to wORLDI, where no relation is protected at all. Passing the relation back to WORLDI gives the two predicates

$$
x \leqslant z \text { if } y>z \text { and } x \leqslant y \text { in WORLDI }
$$

and

$$
y \leqslant z \text { if } x>z \text { and } x>y \text { in WORLDL. }
$$

We must achieve both of these relations. If we achieve the first relation first, we insert the instruction sort $(x z)$ at the beginning of the program, then becuase $x \leqslant z$, the second relation is trivially satisfied. The program obtained is the

$$
\begin{aligned}
& \operatorname{sont}(x z) \\
& \operatorname{sont}(x y) \\
& \operatorname{sont}(y z) .
\end{aligned}
$$

If, on the other hand we try to achieve the second relation first, by inserting the instruction sort $\left(\begin{array}{ll}y & z\end{array}\right)$ at the beginning of the program, the first relation will also be satisfied automatically, and the program will then be

$$
\begin{aligned}
& \operatorname{sont}\left(\begin{array}{ll}
y & z
\end{array}\right) \\
& \operatorname{sont}(x y) \\
& \operatorname{sont}\left(\begin{array}{ll}
y & z
\end{array}\right) .
\end{aligned}
$$

The simultaneous goal problem is essential to all program synthesis: what we have said in this section only begins to explore the subject.

This concludes the presentation of our basic program synthesis techniques. In the next part we will show how the same techniques work together in the synthesis of some more complex examples.

III. PROGRAM SYNTHESIS: THE PATTERN-MATCHER

We will present the synthesis of a simple pattern-matcher to show how the concepts discussed in the previous section can be applied to a non-trivial problem. Later, in Part IV, we shall show how we can con- 
struct an even more complex program, the unification algorithn of Robinson [1965], by modifying the program we are about to synthesize. We must first describe the data structures and primitive operations involved in the pattern-matching and unification problems.

\section{A. Domain and Notations}

The main objects in our domain are expressions and substitutions. 1. Expressions

Expressions are atoms on nested lists of atoms; e.g., (A B (X C) D) is an expression. An atom may be either a variable or a constant. (In our examples we will use $A, B, C, \ldots$ for constants and $U, V, W, \ldots$ for variables.) We have basic predicates atom, var and const to distinguish these objects:

and

$$
\begin{aligned}
& \operatorname{atom}(\ell) \equiv \ell \text { is an atom, } \\
& \operatorname{var}(\ell) \equiv \ell \text { is a variable, } \\
& \text { const }(\ell) \equiv \ell \text { is a constant. }
\end{aligned}
$$

To decompose an expression, we will use the primitive functions head( () and tail ( $\ell)$, defined when $\ell$ is not an atom.

head $(l)$ is the first element of $l$, tail( $(l)$ is the list of all but the first element of $l$.

Thus

$$
\begin{aligned}
& \text { head }((A(X) B) C(D X)))=(A(X) B), \\
& \operatorname{tail}(((A(X) B) C(D x)))=(C(D X)) .
\end{aligned}
$$

We will abbreviate head $(l)$ as $\ell_{1}$ and tail $(\ell)$ as $\ell_{2}$.

To construct expressions we have the concatenation function: if $l$ is any expression and $m$ is a nonatomic expression, $l \cdot m$ is the expression with \& inserted before the first element of $m$. For example

$$
(A(X) B) \cdot(C(D X))=((A(X) B) C(D X))
$$

The predicate occursin $(x)$ is true if $x$ is an atom that occurs in expression $l$ at any Ievel, e.g.,

$$
\text { occursin(A (C (B (A) B) C) is true }
$$

but

$$
\text { occursin }(X Y) \text { is false. }
$$

Finally, we will introduce the predicate constexp(l), which is true if $\ell$ is made up entirely of constants. Thus 


$$
\text { constexp }((A \text { (B) C (DE))) is true }
$$

but

$$
\text { constexp }(X) \text { is false. }
$$

Note that constexp differs from const in that constexp may be true on nonatomic expressions.

\section{Substitutions}

A substitution replaces certain variables of an expression by other expressions. We will represent a substitution as a list of pairs. Thus

$$
(<X(A B)\rangle\langle Y(C Y)\rangle)
$$

is a substitution.

The instantiation function inst (s $l)$ applies the substitution $s$ to an expression $l$. Fon example, if $s$ is the substitution above and $\ell$ is

$$
(X(A Y) X)
$$

then inst $(s \&)$ is

$$
((A B)(A(C Y))(A B)) \text {. }
$$

Note that the substitution is applied by first replacing all occurrences of $X$ simultaneously by (A B) and then all occurrences of $Y$ simultaneously by $(C Y)$. Thus, if the substitution $s$ were

$$
(\langle X Y\rangle\langle Y C\rangle) \text {, }
$$

then inst $(s \&)$ would be

$$
(C(A C) C) \text {. }
$$

The empty substitution $\Lambda$ is represented by the empty list of pairs. Thus, for any expression $\ell$,

$$
\text { inst }(\Lambda \text { \&) }=\ell \text {. }
$$

We regard two substitutions $s_{1}$ and $s_{2}$ as equal (written $s_{1}=s_{2}$ ) if and only if

$$
\text { inst }\left(s_{1} l\right)=\operatorname{inst}\left(s_{2} l\right)
$$

for every expression $\ell$. Thus

and

$$
(\langle X \quad Y>\langle Y \quad C>)
$$

$$
(<\mathrm{X} C\rangle\langle\mathrm{Y} C\rangle)
$$

are regarded as the same substitution.

We can build up substitutions using the functions pair and ${ }^{\circ}$ (composition): If $v$ is a variable and $t$ an expression, pair $(v t)$ is 
the substitution that replaces $v$ by $t$; $i . e .$,

$$
\operatorname{pain}(v t)=(\langle v t\rangle) \text {. }
$$

If $s_{1}$ and $s_{2}$ are two substitutions, $s_{1}{ }^{\circ} s_{2}$ is the substitution with the same effect as applying $s_{1}$ followed by $s_{2}$. Thus

For example, if

$$
\text { inst }\left(s_{1}^{\circ} s_{2} l\right)=\operatorname{inst}\left(s_{2}{ }_{\text {inst }}\left(s_{1} \ell\right)\right) \text {. }
$$

and

$$
s_{1}=(\langle X \quad A\rangle\langle Y B\rangle)
$$

then

$$
s_{2}=\left(\begin{array}{llll}
Z & C\rangle\langle X & D
\end{array}\right)
$$

$$
s_{1}^{\circ} s_{2}=(\langle X \quad A\rangle\langle Y \text { B }\rangle\langle Z C\rangle) \text {. }
$$

Note that for the empty substitution $A$

$$
\Lambda^{\circ} s=s^{0} \Lambda=s
$$

for any substitution $s$.

\section{B. The specifications}

The problem of pattenn-matching may be described as follows. We are given two expressions, pat and arg. Pat can be any expression, but arg is assumed to contain no variables; i.e., constexp(arg) is true. We want to find a substitution $z$ that transforms pat into arg, such that

$$
\text { inst }(\mathrm{z} \text { pat) }=\text { arg. }
$$

We will call such a substitution a match. If no match exists, we want the program to return the distinguished constant NOMATCH. For example, if

and

$$
\text { pat is }(X A(Y \quad B))
$$

$$
\text { ang is }(C A(D B)) \text {, }
$$

we want the program to find the match

$$
(\langle X C\rangle\langle Y D\rangle) \text {. }
$$

On the other hand, if

and

$$
\text { pat is }(X A(X B))
$$

$$
\text { arg is ( } B \text { A (D B ) ), }
$$

then no substitution will transform pat into arg, so the program will yield NOMATCH.

This version of the pattern-matcher is simpler than the patternmatching algorithms usually implemented in programing languages because of the absence of "sequence" or "fragment" variables. Our variables must match exactly one expression, whereas a fragment variable may match any number of expressions. Because of 
the absence of fragment variables, a match, if it exists, will be unique. Thus if

$$
\begin{aligned}
& \text { pat is }(X Y Z) \text { and } \\
& \text { arg is }((A B) C(A B)) \text {, }
\end{aligned}
$$

$X$ and $Z$ must be bound to ( $A B$ ), and $Y$ must be bound to $C$. If

$$
\begin{aligned}
& \text { pat is }(X Y) \text { and } \\
& \text { arg is }(A B C) \text {, }
\end{aligned}
$$

no match is possible at all. (If $X$ and $Y$ were fragment variables, four matches would be possible.)

In mathematical notation the specifications for our pattem-matcher are:

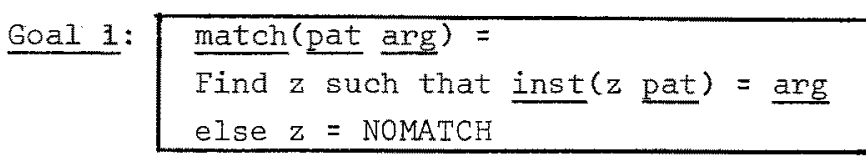

where "Find $z$ such that $P(z)$ else $Q(z)$ " means find a $z$ such that $P(z)$ if one exists; otherwise, find a $z$ such that $Q(z)$.

The above specifications do not completely capture our intentions; for instance, if

$$
\begin{aligned}
& \text { pat is }(X Y) \text {, and } \\
& \underline{\arg } \text { is }(A B) \text {, }
\end{aligned}
$$

then the substitution

$$
z=(\langle X A\rangle\langle Y \quad B\rangle\langle Z C\rangle)
$$

will satisfy oun specifications as well as

$$
z=(\langle X \quad A\rangle\langle Y \quad B\rangle) \text {. }
$$

We have neglected to include in our specifications that no substitutions should be made for variables that do not occur in pat. We will call a match that satisfies this additional condition a most general match.

An interesting characteristic of the synthesis we present is that even if the user forgets to require that the match found be most general, the system will be able to strengthen the specifications automatically to imply this condition, using the method outlined in section II-D. Therefore we will begin the synthesis using the weaker specifications.

\section{The Synthesis: The Base Cases}

Rather than listing all the knowledge we require in a special sec- 
tion at the beginning, we will mention a rule only when it is about to be used. Funthermore, if a rule seems excessively trivial we will omit it entirely. The general strategy is to first work on

Goal 2: Find z such that inst $(z$ pat $)=$ arg.

If this is found to be impossible (i.e., if it is proven that no such $z$ exists), we will work on

Goal 3: Find a $z$ such that $z=$ NOMATCH;

which is seen to be trivially satisfied by taking $z$ to be NOMATCH.

Thus, from now on we will be working primarily on Goal 2. However, in working on any goal we devote a portion of our time to showing that the goal is impossible to achieve. When we find cases in which Goal 2 is proven impossible, we will automatically return NOMATCH, which satisfies Goal 3 .

We have in oun knowledge base a number of rules concerning inst, including

Rule I: $\quad$ inst $(s x)=x$ for any substitution $s$

$$
\text { if } \operatorname{constexp}(x)
$$

Rule 2: $\quad$ inst $($ pair $(v t) v)=t$

$$
\text { if } \operatorname{vax}(v)
$$

We assume that these rules are retrieved by pattern-directed function invocation on Goal 2. Rule 1 applies only in the case that constexp (pat) and pat $=$ arg. We cannot prove either of these conditions; their truth on falsehood depends on the particular inputs to the program. We use these predicates as conditions for a hypothetical world-split. In the case that both of these conditions are true, Rule I tells us that any substitution is a satisfactory match. We will have occasion to tighten the specifications of our program later; as they stand now, we will simply return ztany. The portion of the program we have constructed so far reads

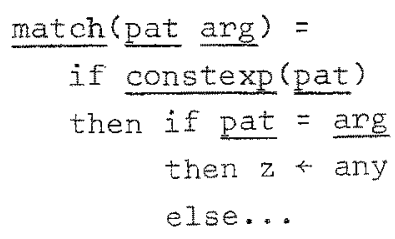

On the other hand, in the case constexp (pat) and pat $\neq$ arg, Rule $I$ 
tells us that

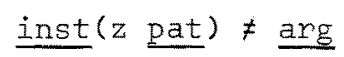

for any $z$. Hence we are led to try to satisfy Goal 3 and take $z+$ NOMATCH.

We now consider the case

$$
\sim \text { constexp (pat). }
$$

Ruel 2 establishes the subgoal

$$
\text { var (pat). }
$$

This is another occasion for a hypothetical world-split. When var(pat) is true, the program must return pair(pat arg); the program we have constructed so far is

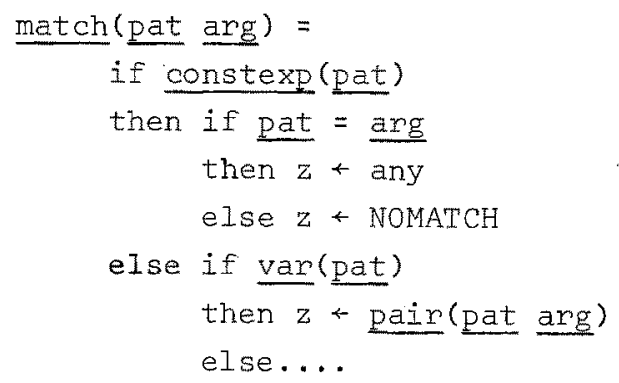

Hencefore we assume $\operatorname{var}(p a t)$. Recall that we have been assuming also that constexp (pat). To proceed we make use of the following additional knowledge about the function inst:

Rule 3: $\quad$ inst $(s x \cdot y)=$ inst $(s x)$. inst $(s y)$ for any substitution $s$. This rule applies to our Goal 2 if pat $=x \cdot y$ for some expressions $x$ and $y$. We have some additional knowledge about expressions in general:

Rule 4: $u=u_{1} \cdot u_{2}$ if $\sim \operatorname{aton}(u)$

Recall that $u_{1}$ is an abbreviation for head(u) and $u_{2}$ is an abbreviation for tail $(u)$.

Rule 5: $u \neq v \cdot w$ for any $u, v$, and $w$ if $\operatorname{atom}(u)$

Using Rule 4, we generate a subgoal

$\sim$ atom(pat).

Since we have already assumed $\sim$ constexp (pat) and $\sim \operatorname{var}($ pat), we can actually prove atom(pat) using knowledge in the system. Therefore pat=pat 1 . pat 2 and using Rule 3 our Goal 2 is then 
reduced to

Goal 4: Find $z$ such that inst $(z$ pat 1$)$ inst $\left(z\right.$ pat $\left._{2}\right)=$ arg. We now make use of some general list-processing knowledge.

Rule 6: To prove $x \cdot y=u \cdot v$, prove $x=u$ and $y=v$. Applying this rule, we generate a subgoal to show that

$$
\underline{\arg }=\mathrm{u} \cdot \mathrm{v}
$$

for some $u$ and $v$. Applying Rule 4, we know this is true with

$u=\underline{a r g}_{1}$ and $v=\underline{a r g}_{2}$ if

$$
\sim \text { atom (arg). }
$$

This is another occasion for a hypothetical world-split.

Thus, by Rule 6 , in the case that atom(ang), our subgoal reduces to

Goal 5: Find $z$ such that

$$
\text { and } \begin{aligned}
& \text { inst }\left(z \mathrm{pat}_{1}\right)=\text { arg }_{1} \\
& \text { inst }\left(z \mathrm{pat}_{2}\right)=\mathrm{arg}_{2} \text {. }
\end{aligned}
$$

We will portpone treatment of this goal until after we have considered the other case, in which

\section{atom (arg)}

holis. In this case Rule 5 tells us that

$$
\text { inst }\left(z \underline{p a t}_{1}\right) \cdot \text { inst }\left(z \underline{p a t}_{2}\right) \neq \text { arg }
$$

for any $z$. Hence, our goal is unachievable in this case, and we can take

$$
z+\text { NOMATCH. }
$$

The program so far is

$$
\begin{aligned}
& \text { match (pat arg) }= \\
& \text { if constexp (pat) } \\
& \text { then if pat }=\frac{\text { arg }}{\text { if }} \\
& \text { then } z+\text { any } \\
& \text { else } z+\text { NOMATCH } \\
& \text { else if var }(\text { pat) } \\
& \text { then } z+\text { pair }(\text { pat } \text { arg) } \\
& \text { else if atom (arg) } \\
& \text { then } z+\text { NOMATCH } \\
& \text { else ... }
\end{aligned}
$$

For the as yet untreated case neither pat nor arg is atomic. Henceforth using Rule 4 we assume that pat is pat ${ }_{1}$ pat $_{2}$ and ang is $\underline{\arg }_{1} \cdot \underline{\arg }_{2}$. 


\section{The Synthesis: The Inductive Case}

We will describe the remainder of the synthesis in less detail, because the reader has already seen the style of reasoning we have been using. Recall that we had postponed our discussion of Goal 5 in order to consider the case in which arg is atomic. Now that we have completed our development of that case, we resume our work on Goal 5 :

$$
\begin{aligned}
& \text { Find } z \text { such that } \\
& \text { inst }\left(z \mathrm{pat}_{1}\right)=\mathrm{ang}_{1}, \text { and } \\
& \underline{\text { inst }}\left(\mathrm{z} \mathrm{pat}_{2}\right)=\mathrm{ang}_{2} .
\end{aligned}
$$

This is a conjunctive goal, and is treated analogously to the goal in the simultaneous linear equations example: each conjunct is treated separately. The system will attempt to use a recursive call to the pattem-matcher itself in solving each conjunct.

The interaction between the two conjuncts is part of the challenge of this synthesis. It is quite possible to satisfy each conjunct separately without being able to satisfy them both together. For

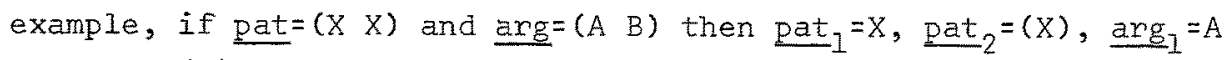
and $\arg _{2}=(B)$. Thus $z=(\langle X \quad A\rangle)$ satisfies the first conjunct, $z=(\langle X \quad B\rangle)$ satisfies the second conjunct, but no substitution will satisfy both conjuncts because it cannot match $X$ against both $A$ and $B$. Some mechanism is needed to ensure that the expression assigned to a variable in solving the first conjunct is the same as the expression assigned to that variable in solving the second conjunct.

There are several ways to approach this difficulty. For instance, the programmen may satisfy the two conjuncts separately and then attempt to combine the two substitutions thereby derived into a single substitution. Or he may actually replace those variables in pat 2 that also occur in $\mathrm{pat}_{1}$ by whatever expressions they have been matched against, before attempting to match pat $\underline{\mathrm{s}}_{2}$ against $\underline{\mathrm{arg}}_{2}$. or he may simply pass the substitution that satisfied the first conjunct as a third argument to the pattern-matcher in working on the second conjunct. The pattem-matcher must then check that the matches assigned to variables are consistent with the substitution given as the third argument. 
We will examine in this section how a system would discover the second of these methods and consider in a later section how the third method could be discovered. We will not consider the first method here because it is not easily adapted to the unification problem.

Our strategy for approaching the conjunctive goal is as follows. We will consider the first conjunct independently:

Goa] 6: Find $z$ such that inst $\left(z\right.$ pat $\left._{1}\right)=$ arg $_{I}$.

If we find a $z$ that satisfies this goal, we will substitute that $z$ into the second conjunct, giving

Goal 7: Prove inst $\left(z \underline{\text { pat }}_{2}\right)=$ arg $_{2}$.

If we are successful in Goal 7, we are done; however, if we fail, we will try to generalize $z$. In other words, we will try to find a broaden class of substitutions that satisfy Goal 6 and from these select one that also satisfies Goal 7. This is the method we introduced to solve conjunctive goals in section II-E. Applying this strategy, we begin work on Goal 6. We first use a rule that relates the construct

Find $z$ such that $P(z)$

to the construct

Find $z$ such that $P(z)$ else $Q(z)$.

Rule 7: To find $z$ such that $P(z)$,

find $z_{1}$ such that $P\left(z_{1}\right)$

else $Q\left(z_{1}\right)$ for some predicate $Q$,

and prove $\sim Q\left(z_{1}\right)$.

This rule, applied to Goal 6 causes the generation of the subgoal Goal 8: Find $z_{1}$ such that inst $\left(z_{1} \underline{\text { pat }}_{1}\right)=\arg _{1}$ else $Q\left(z_{I}\right)$, and prove $\sim Q\left(z_{1}\right)$.

This subgoal matches the top-level Goal 1 , where $Q\left(z_{1}\right)$ is $z_{1}=$ NOMATCH. This suggests establishing a recursion at this point, taking

$z_{I}=$ match (pat arg $\left._{1}\right)$

Temination is easily shown, because both pat and $_{1} \mathrm{ang}_{I}$ are proper subexpressions of pat and arg, respectively. It remains to show, according to Rule 7 , that $z_{1} \neq$ NOMATCH. This causes another 
hypothetical world-split: in the case $z_{I}=$ match $\left(\operatorname{pat}_{1} \arg _{1}\right)=$ NOMATCH (i.e., no substitution will cause pati and arg to match), we can show that no substitution can cause pat and arg to match

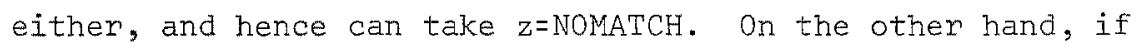
match (pat $\underline{\mathrm{arg}}_{1}$ ) $\neq$ NOMATCH, we try to show Goal 7 , i.e., inst $\left(z_{1} \underline{\text { pat }}_{2}\right)=\underline{\arg }_{2}$.

However, we fail in this attempt; in fact we can find sample inputs pat and arg that provide a counter-example to Goal 7 (e.g., pat $\left.=(A X), \arg =(A B), z_{1}=\Lambda\right)$. Thus we go back and try to generalize our solution to Goal 6 .

We already have a solution to Goal 6: we know $\underline{i n s t}_{1} \mathrm{z}_{1}$ pat $\left._{1}\right)=$ arg $_{1}$. We also can deduce that constexp $\left(\arg _{1}\right)$, because we have assumed constexp (arg). Hence Rule 1 tells us that

$$
\text { inst }\left(w \mathrm{arg}_{1}\right)=\underline{\arg }_{1}
$$

for any substitution $w$. Hence

i.e.,

$$
\text { inst }\left(w \operatorname{inst}\left(z_{1} \text { pat }_{1}\right)\right)=\text { arg }_{I} \text {, }
$$

$$
\operatorname{inst}\left(z_{1}^{o} \mathrm{pat}_{I}\right)=\arg _{I}
$$

for any substitution $w$. Thus having one substitution $z_{1}$ that satisfies Goal 6, we have an entire class of substitutions, of form $z_{l}{ }^{\circ}$, that also satisfy Goal 6 . These substitutions may be considered to be "extensions" of $z_{1}$; although $z_{1}$ itself may not satisfy Goal 7 , perhaps some extension of $z_{1}$ will.

The above reasoning is straightforward enough to justify, but further work is needed to motivate a machine to pursue it.

It remains now to find a single $w$ so that ${ }^{z_{1}}{ }^{\circ} \mathrm{W}$ satisfies Goal 7 , i.e., Goal 9: Find w such that inst $\left(z_{1}{ }^{\circ} \mathrm{w} \underline{\text { pat }}_{2}\right)=\underline{a r g}_{2}$, or equivalentiy,

Find w such that inst (w inst $\left.\left(z_{1} \underline{\text { pat }}_{2}\right)\right)=\underline{\arg }_{2}$. applying Rule 7 , we establish a new goal

Goal 10: Find w such that inst $\left(w\right.$ inst $\left.\left(z_{1} \underline{\text { pat }}_{2}\right)\right)=\underline{\arg }_{2}$

else $Q(w)$, and prove $\sim Q(w)$

This goal is an instance of our top-level goal, taking pat to be

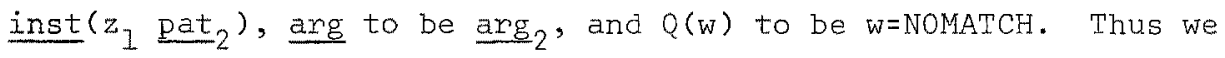
attempt to insert the recursive call 


$$
z_{2} \leftarrow \operatorname{match}\left(\text { inst }\left(z_{1} \quad \operatorname{pat}_{2}\right) \arg _{2}\right)
$$

into our program at this point and take $w$ to be $z_{2}$. However, we must first establish

i.e.s

$$
\sim Q\left(z_{2}\right) \text {, }
$$

$$
\text { match(inst } \left.\left(z_{1} \underline{\text { pat }}_{2}\right) \underline{\arg }_{2}\right) \neq \text { NOMATCH. }
$$

We cannot prove this. We have counter-examples, e.g., if $\underline{\text { pat }}=(A A), \underline{\arg }=(A B)$ and $z_{I}=\Lambda$,

then

$$
\text { match(inst }(\Lambda \text { A) B) = NOMATCH. }
$$

Therefore we split on this condition.

In the case

$$
\text { match (inst } \left.\left(z_{1}{\underline{p^{2}}}_{2}\right){\underline{\arg _{2}}}_{2}\right) \neq \text { NOMATCH }
$$

Goal 10 is satisfied. Thus $w=z_{2}$ also setisfies Goal 9 , and $z=z_{1}{ }^{\circ} z_{2}$ satisfies Goal 7 .

our program so far is

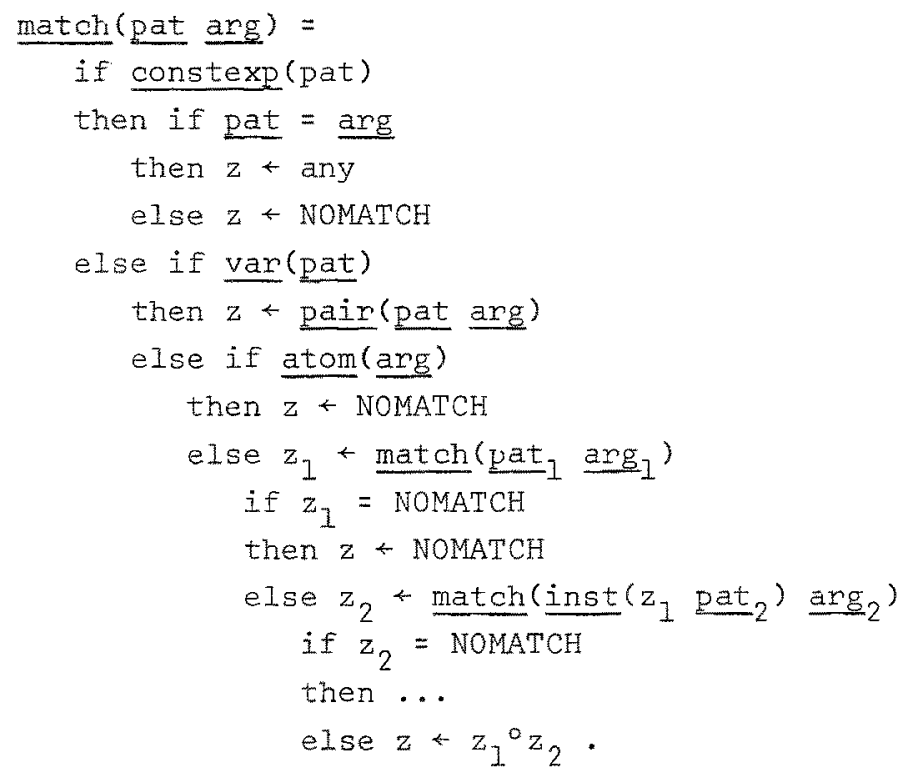

E. The Synthesis: The Strengthening of the Specifications

We have gone this far through the synthesis using the weak specifications, i.e., without requiring that the match found be most general. In fact, the match found may or may not be most general 
depending on the value taken for the unspecified substituion "any" produced in the very finst case. The synthesis is nearly complete. However, we will be unable to complete it without strengthening the specifications and modifying the program accordingly. We now have only one case left to consider. This is the case in which $z_{2}=$ NOMATCH

i.e.,

match $\left(\underline{\text { inst }}\left(\mathrm{z}_{1} \underline{\text { pat }}_{2}\right)=\right.$ NOMATCH.

This means that no substitution w satisfies

or, equivalently

$$
\underline{\text { inst }}\left(w \underline{\text { inst }}\left(z_{1} \text { pat }_{2}\right)\right)=\underline{\text { arg }}_{2} \text {, }
$$

inst $\left(z_{1}{ }^{\circ} \mathrm{w}\right.$ pat 2$) \neq \underline{a r g}_{2}$ for every substitution $w$.

This means that no substitution of form $z_{1}{ }^{\circ}$ w could possibly satisfy inst $\left(z_{1}{ }^{\circ} \mathrm{W}\right.$ pat $)=$ arg.

We here have a choice: we can try to find a substitution s not of form $z_{1}{ }^{\circ} \mathrm{w}$ that satisfies

$$
\text { inst }\left(s \text { pat }_{1}\right)=\underline{\arg }_{1}
$$

and repeat the process; or we could try to show that only a substitution $s$ of form $z_{1}{ }^{\circ} \mathrm{w}$ could possibly satisfy

$$
\text { inst }\left(s \underline{\text { pat }}_{1}\right)=\underline{a r g}_{1} \text {, }
$$

and therefore we can take $\mathrm{z}=$ NOMATCH.

We have already extended the class of substitutions that satisfy the condition once; therefore we pursue the latter course. We try to show that the set of substitutions $s=z_{1}{ }^{\circ} \mathrm{W}$ is the entire set of solutions to

$$
\text { inst (s } \left.\text { pat }_{1}\right)=\underline{\text { ang }}_{1} \text {. }
$$

In other words, we show that for any substitution $s$,

$$
\text { if inst }\left(s \mathrm{pat}_{I}\right)=\arg _{I} \text { then } s=z_{I}{ }^{\circ} \mathrm{W} \text { for some } \mathrm{w} \text {. }
$$

This condition is equivalent to saying that $z_{1}$ is a most general match. We cannot prove this about $z_{I}$ itself; however, since $z_{1}$ is match (pat $\underline{a r g}_{1}$ ) it suffices to add the condition to the specifications for match, as described in Section II-D. The strengthened specifications now read

Find $z$ such that

$$
\begin{aligned}
& \text { (inst }(z \text { pat) }=\text { arg and } \\
& \text { for all s [if inst (s pat) = arg } \\
& \text { then } \left.\left.s=z^{\circ} w \text { for some } w\right]\right\} \\
& \text { else } z=\text { NOMATCH. }
\end{aligned}
$$


Once we have strengthened the specifications it is necessary to go through the entire program and see that the new, stronger specifications are satisfied, modifying the program if necessary. In this case no major modifications are necessary; however, the assignment

$$
z+\text { any }
$$

that occurs in the case in which pat and arg are equal and constant is further specified to read

$z \leftarrow \Lambda$.

Our final program is therefore

match (pat $\underline{\arg })=$

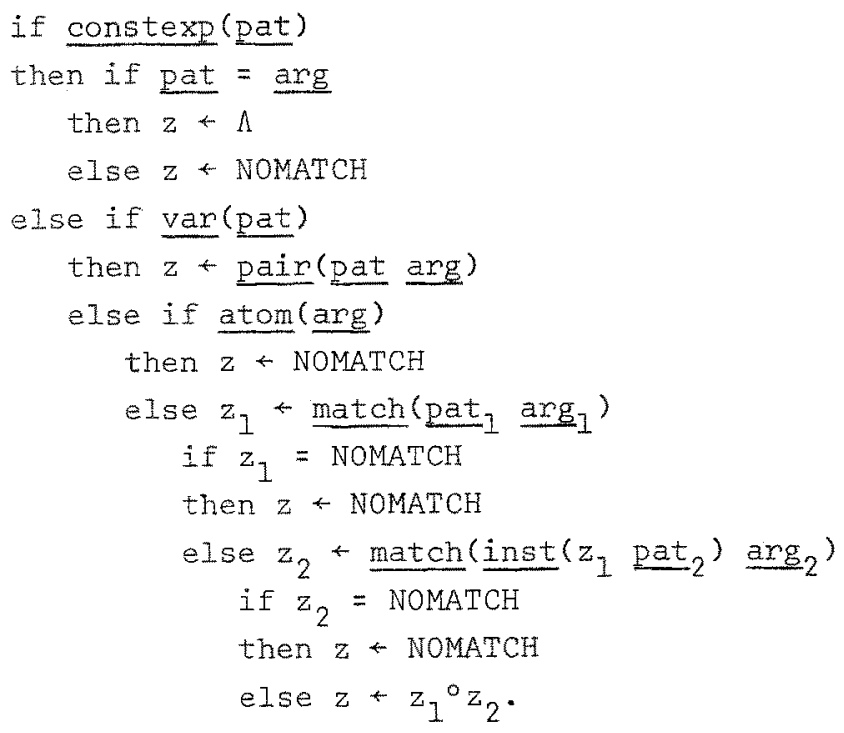




\section{F. Alternative Programs}

The above pattem-matchen is only one of may pattern-matchers that can be derived to satisfy the same specifications. In pursuing the synthesis the system has made many choices; some of the altemative paths result in a failure to solve the problem altogether, whereas other paths result in different, possibly better programs. In this section we will indicate how a system might make a different decision in the above synthesis and, consequently, drive a different program.

In the above synthesis we derived a goal (Goal 9):

Find $w$ such that inst $\left(z_{1}^{\circ}{ }_{\mathrm{w}} \underline{\mathrm{pat}}_{2}\right)=\underline{\mathrm{arg}}_{2}$.

We chose at that point to transform the goal to the equivalent

Find $w$ such that inst $\left(w\right.$ inst $\left.\left(z_{1} \underline{p a t}_{2}\right)\right)=\underline{\arg }_{2}$,

and then apply Rule 7 , which added an else-clause to the goal, yielding Goal 10. We then matched Goal 10 against our top-level goal, introducing the second recursive call into our program. It would be equally plausible that the system should apply Rule 7 directly to Goal 9 , giving a new goal

Goal 10': Find w such that inst $\left(z_{1}{ }^{\circ} \underline{\text { pat }}_{2}\right)=\underline{a r g}_{2}$ else $Q(w)$, and prove $\sim(w)$.

The system may now attempt to use a recursive call to satisfy Goal 10'. However, this goal is not a precise instance of our top-level goal; we are demanding not only that a match be found between pat $_{2}$ and $\underline{\text { ang }}_{2}$, but also that the match be of form $\mathrm{z}_{1}{ }^{\circ} \mathrm{W}$, where $z_{1}$ is the output of the previous recursive call. We must therefore generalize our program to take three inputs: pat, arg, and alist. We insist that the match found be an extension of alist. In the case that alist is $\Lambda$, the new program will be identical to the old one. On the other hand, if alist $=z_{1}$, pat $=\underline{p a t}_{2}$ and $\underline{a r g}=\underline{a r g}_{2}$, a recursive call to the new program suffices to satisfy Goal 10'.

In mathematical notation, the stronger specifications now read

$$
\begin{aligned}
& \text { Find } z \text { such that }\left[\frac{\text { inst }(z \text { pat })}{z=\text { alisto }_{w}} \text { for some } w\right] \\
& \text { else } z=\text { NomATCH. }
\end{aligned}
$$


We will call the generalized program

\section{match2 (pat arg alist).}

The original program match will be written as a call to the more general auxiliary program:

$$
\text { match }(\text { pat } \text { arg })=\operatorname{match} 2(\text { pat } \text { ang } \Lambda) \text {. }
$$

The portion of the program already constructed in the synthesis of match must be systematically modified to satisfy the strengthened requirements of match2.

First, in the case that constexp(pat) where pat=arg, we originally took z any. However, we must now take

$$
z \leftarrow \text { alist }^{\circ} \text { any, }
$$

becuase the substitution found must be an extension of alist.

In the case that var(pat) we originally took $z$ pair (pat arg); however, here we must check to see whether pat has already been matched ageinst something other than arg. The new program segment is

$$
\begin{aligned}
& \text { if inst (alist pat) }=\text { pat } \\
& \text { then } z+\text { alistopair (pat arg) } \\
& \text { else if inst (alist pat) }=\text { arg } \\
& \text { then } z \leftarrow \text { alist } \\
& \text { else } z \leftarrow \text { NoMATCH. }
\end{aligned}
$$

of course.we are omitting the details of synthesis; the actual derivation is somewhat more lengthy.

The call $z_{1} \leftarrow$ match $\left(\right.$ pat $\left._{I} \frac{\text { arg }_{1}}{}\right)$ must be replaced by

$$
z_{1} \leftarrow \text { match2 (pat } 1 \text { arg } 1 \text { alist). }
$$

Having modified the portion of the program already constructed, the system continues the synthesis. A recursive call

$$
z_{2}+\operatorname{match}_{2}\left(\operatorname{pat}_{2} \arg _{2} z_{1}\right)
$$

satisfies Goal 9; the balance of the synthesis parallels the development of the first program, including the further strengthening of the specifications to ensure that the match found is the most general possible.

The value of the second recursive call is shown to satisfy the strengthened top-level goal.

The program derived from this alternative synthesis is 


\section{$\underline{\operatorname{match}}(\underline{p a t} \underline{\arg })=\underline{\operatorname{match} 2}($ pat $\underline{\arg } \Lambda)$}

where

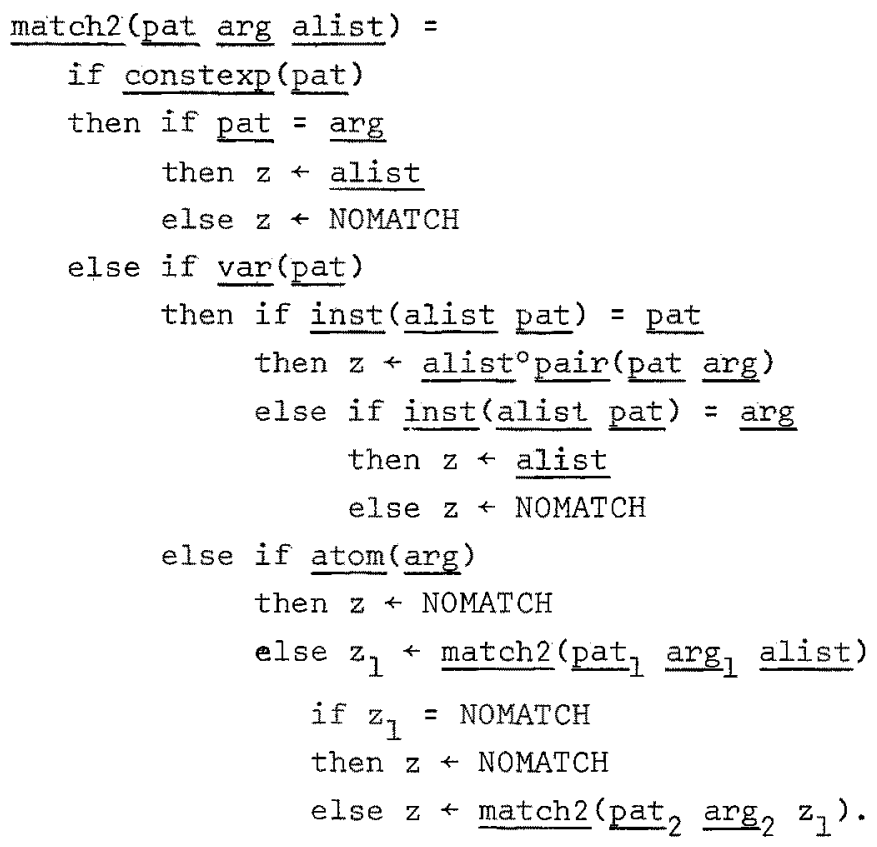

IV. PROGRAM MODIFICATION: THE UNIFICATION ALGORITHM

In general, we cannot expect a system to synthesize an entire complex program from scratch, as in the pattern-matcher example. We would like the system to remember a large body of programs that have been synthesized before and the method by which they were constructed. When presented with a new problem, the system should check to see if it has solved a similar problem before. If so, it may be able to adapt the technique to the old program to make it solve the new problem.

There are several difficulties involved in this approach. First, we cannot expect the system to remember every detail of every synthesis in its history. Therefore, it must decide what to remember and what to forget. Second, the system must decide which problems are similar to the one being considered, and the concept of similarity is somewhat ill-defined. Third, having found a similar program, the system must somehow modify the old synthesis to solve the new problem. We will concentrate only on the latter of these 
problems in this discussion. We will illustrate a technique for prognam modification as appiled to the synthesis of a version of Robinson's unification algorithm.

\section{A. The Specifications}

Unification may be considered to be a generalization of pattern matching in which variables appear in both pat and arg. The problem is to find a single substitution (called a "unifier") that, when applied to both pat and arg, will yield identical expressions. for instance, if

$$
\text { pat }=(X A)
$$

and

$$
\underline{\arg }=(B Y) \text {, }
$$

then a possible unifier of pat and arg is

$(<X \quad B>\langle Y \quad A>)$.

The close analogy between pattern-matching and unification is clear. If we assume that the system remembers the patternmatcher we constructed in Sections III-2 through III-5 and the goal structure involved in the synthesis, the solution to the unification problem is greatly facilitated.

The specifications for the unification algonithm, in mathematical notation, are

unify (pat arg $)=$

Find $z$ such that inst $(z$ pat $)=$ inst $(z$ arg $)$

else $z=$ NOMATCH

3. The Analogy with the Pattern-Matcher

For purposes of comparison we rewrite the match specifications: match (pat $\underline{\text { arg }})=$

Find $z$ such that inst $(z \underline{\text { pat }})=\underline{\text { arg }}$

eIse $z=$ NOMATCH.

In formulating the analgy, we identify unify with natch, pat with pat, the arg in unify (pat arg) with arg, and inst ( $\mathrm{z}$ arg) also with arg. In accordance with this analogy, we must systematically alter the goal structure of the pattern-matcher synthesis. For example, Goal 5 becomes modified to read 


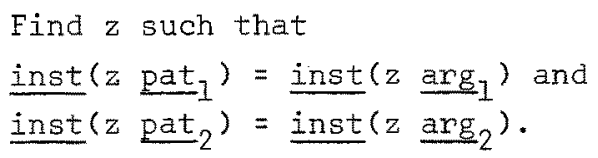

In constructing the pattern-matcher, we had to break down the synthesis into various cases. We will try to maintain this case structure in formulating our new program. Much of the savings derived from modifying the pattern-matcher instead of constructing the unification algorithm from scratch arises because we do not have to deduce the case splitting all over again.

A difficult step in the pattern-matcher synthesis involved the strengthening of the specifications for the entire program. We added the condition that the match found was to be "most general." In formulating the unification synthesis, we will immediately strengthen the specifications in the analogous way. The strengthened specifications read

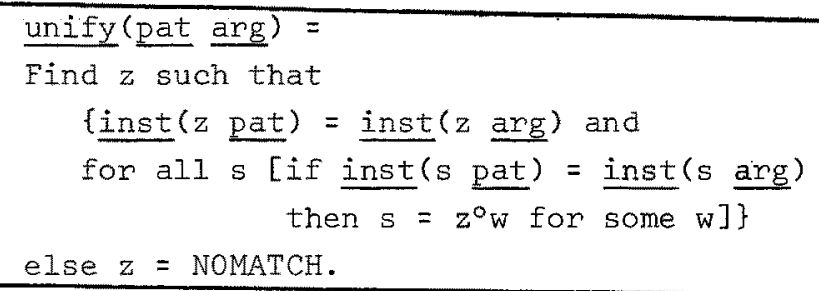

Following Robinson [1965], we will refer to a unifier satisfying the new condition as a "most general unifier."

Note that this alteration process is purely syntactic; there is no reason to assume that the altered goal structure corresponds to a valid line of reasoning. For instance, simply because achieving Goal 2 in the pattern-matching program is useful in achieving Goal 1 does not necessarily imply that achieving Goal $2^{\prime}$ in the unification algorithm will have any bearing on Goal I'. The extent to which the reasoning carries over depends on the soundness of the analogy. If a portion of the goal structure proves to be valid, the corresponding segment of the program can still remain; otherwise, we must construct a new program segment.

\section{The Modification}

Let us examime the first two cases of the unification synthesis in full detail, so that we can see exactly how the modification 
process works. In the pattern-macher, we generated the subgoal (Goal 2)

Find $z$ such that inst $(\mathrm{z}$ pat) $=$ arg.

The corresponding unification subgoal is

Find $z$ such that inst $(z$ pat $)=\operatorname{inst}(z$ arg $)$.

In the pattern-matcher we first considered the case constexp (pat) where pat=ang. In this case the corresponding program segment is $z+\Lambda$.

This segment also satisfies the modified goal in this case, because inst $(\Lambda$ pat $)=\underline{\text { inst }}(\Lambda$ arg $)$.

The system must also check that $\Lambda$ is a most general unifier, i.e., for any $s$ [if inst (s pat) = inst (s arg) then $s=\Lambda^{\circ} \mathrm{W}$ for some $\left.W\right]$.

This condition is easily satisfied, taking $w=s$. Thus, in this case, the program segment is correct without any modification. The next case does require some modification. In the patternmatcher, when constexp (pat) is true and patfarg, $z$ is taken to be NOMATCH. However, in this case in the unification algorithm we must check that

i.e. inst (s pat) $\neq$ inst (s arg),

\section{pat inst (s arg)}

for any $s$, in order to take $z=N O M A T C H$. Since for unification arg may contain variables, this condition cannot be satisfied. We must therefore try to achieve the specifications in some other way. In this case (where constexp (pat)), the specifications of the unification algorithm reduce to

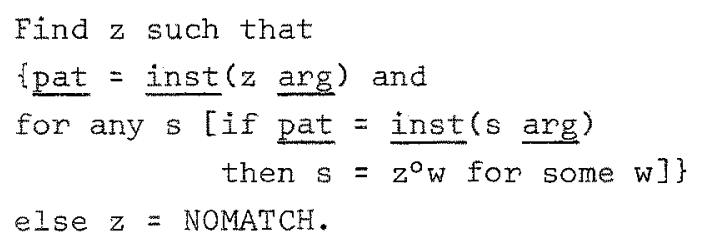

These specifications are precisely the specifications of the pattern-matcher with pat and arg reversed; consequently, we can invoke match(arg pat) at this point in the program.

The balance of the modification can be carried out in the same manner. The derived unification algorithm is 


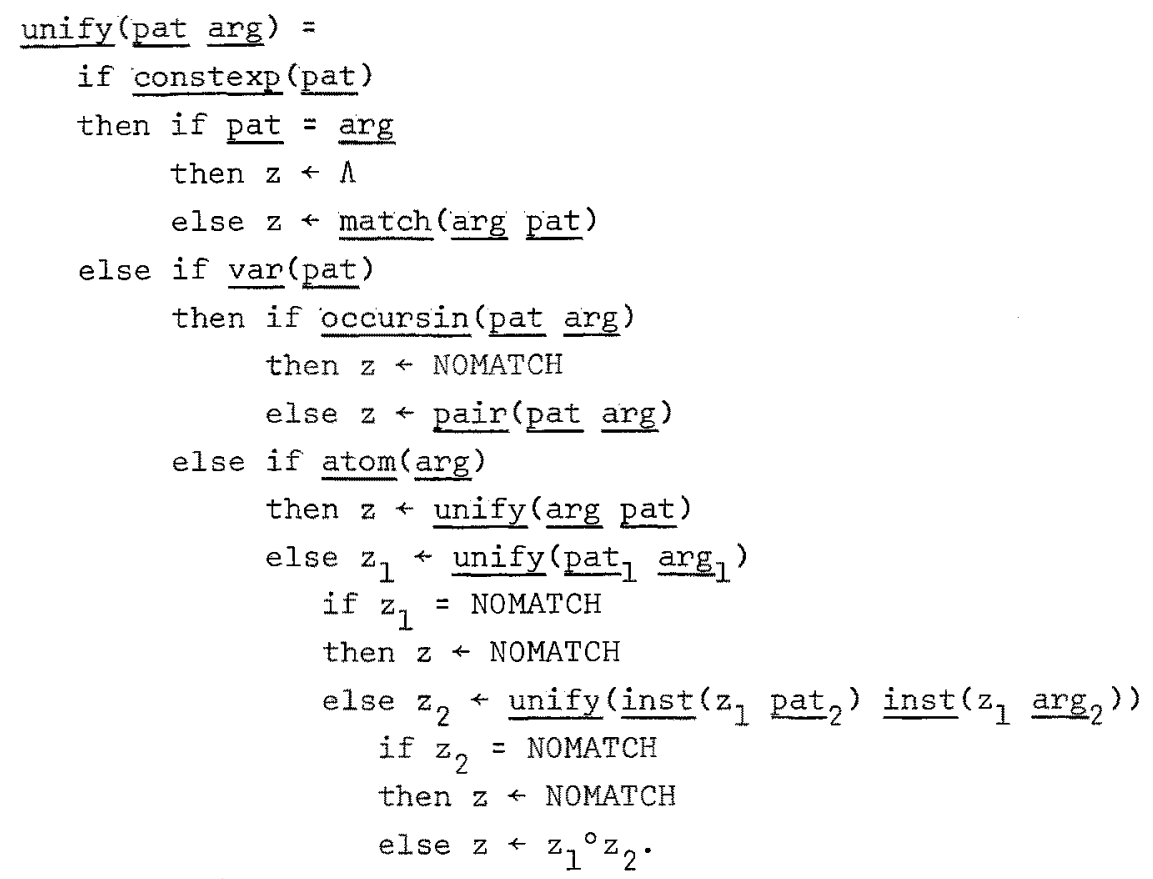

Recall that occursin(pat arg) means that pat occurs in arg as a subexpression.

The termination of this program is considerably more difficult to prove than was the termination of the pattern-matcher. However, the construction of the unification algorithm from the pattern-matcher is much easier than the initial synthesis of the pattern-matcher itself.

Note that the program we have constructed contains a redundant branch. The expression

$$
\begin{aligned}
& \text { if pat }=\underline{\text { arg }} \\
& \text { then } z+\Lambda \\
& \text { else } z+\text { match (arg pat) }
\end{aligned}
$$

could be reduced to

$$
z+\text { match (arg pat). }
$$

Such improvements would not be made until a later optimization phase. 


\section{v. DISCUSSION}

\section{A. Implementation}

Implementation of the techniques presented in this paper is underway. Some of them have already been implemented. Others will require further development before an implementation will be possible. We imagine the rules, used to represent reasoning tactics, to be expressed as programs in a PLANNER-type language. Oun own implementation is in QLISP (Reboh and Sacerdoti [1973]). Rules are summoned by pattern-directed function invocation.

Worlds have been implemented using the context mechanism of QLISP, which was introduced in QA4 (Rulifson et a1. [1972]). The controlstructure necessary for the hypothetical worlds, which involve an actual splitting of the control path as well as the assertional. data base, is expressed using the multiple environments (Bobrow and Wegbreit [1973]) of INTERLISP (Teitelman [1974]). The hypothetical world-splitting has been implemented, but we have yet to experiment with the various strategies for controlling it.

The existing system is capable of producing simple programs such as the union function, the program to sort two variables from Part II, or the 10op-free segments of the pattern-matcher from Part III.

The generalization of specifications (Sections II-4 and III-5) is a difficult technique to apply without its going astray. We will develop heuristics to regulate it in the course of the implementation. Similarly, our approach to conjunctive goals (Section II-5) needs further explication.

\section{B. Historical Context and Contemporary Research}

Early work in program synthesis (e.g. Simon [1963], Green [1969], Waldinger and Lee [1969]), was limited by the problem-solving capabilities of the respective formalisms involved (the General Problem Solver in the case of Simon, resolution theorem proving in the case of the others). Our paper on loop formation (Manna and Waldinger [1971]) was set in a theorem-proving framework, and paid little attention to the implementation problems. 
It is typical of contemporary program synthesis work not to attempt to restrict itself to a formalism; systems are more likely to write programs the way a human programmer would write them. For example, the recent work of Sussman [1973] is modelled after the debugging process. Rather than trying to produce a correct program at once, Sussman's system rashly goes ahead and writes incorrect programs which it then proceeds to debug. The work reported in Green et al. [1974] attempts to model a very experienced programmer. For example, if asked to produce a sort program, the system recalls a variety of sorting methods and asks the user which he would like best.

The work reported here emphasizes reasonging more heavily than the papers of Sussman and Green. For instance, in our synthesis of the pattern-matcher we assumed no knowledge about patternmatching itself. Thus our system would be unlikely to ask the user what kind of pattern-matcher he would like. Of course we do assume extensive knowledge of lists, substitutions, and other aspects of the subject domain.

Although Sussman's debugging approach has influenced our treatment of program modification and the handling of simultaneous goals, we tend to rely more on logical methods than Sussman. Furthermore, Sussman deals only with programs that manipulate blocks on a table; therefore he has not been forced to deal with problems that are more crucial in conventional programing, such as the formation of conditionals and loops.

The work of Buchanan and Luckham [1974] (see also Luckham and Buchanan [1974]) is closest to ours in the problems it addresses. However, there are differences in detail between our approach and theirs:

The Buchanan-Luckham specification language is first-order predicate calculus; ours allows a variety of other notations. Their method of forming conditionals involves an auxiliary stack; ours uses contexts and the Bobrow-Wegbreit control structures. In the Buchanan-Luckham system the loops in the program are iterative, and are specified in advance by the user as "iterative rules," whereas in our system the (recursive) loops are introduced by the system itself when it recognizes a relationship between the 
top-level goal and a subgoal. The treatment of programs with side effects is also quite different in the Buchanan-Luckham system, in which a model of the world is maintained and updated, and assertions are removed when they are found to contradict other assertions in the model. Our use of contexts allows the system to recall past states of the world and avoids the tricky problem of determining when a model is inconsistent. I should be added that the implenentation of the Buchanan-Luckham system is considerably more advanced than ours.

\section{Conclusions and Future Work}

We hope we have managed to convey in this paper the promise of program synthesis, without giving the false impression that automatic synthesis is likely to be immediately practical. A computer systen that can replace the human programmer will very likely be able to pass the rest of the Turing test as well.

Some of the approaches to program synthesis that we feel will be most fruitful in the future have been given little emphasis in this paper because they are not yet fully developed. For example, the technique of program modification, which occupied only one small part of the current paper, we feel to be central to future program synthesis work. The retention of previously constructed programs is a powerful way to acquire and store knowledge. Furthermore program optimization and program debugging are just special cases of program modification.

Another technique that we believe will be valuable is the use of more visual or graphic representations, that convey more of the properties of the object being discussed in a single structure. For example, we have found that the synthesis of the pattern matcher could be made shorter and more intuitive by the introduction of the substitution notation of mathematical logic. If we represent an expression $P$ as $P\left(x_{1}, \ldots, x_{n}\right)$, where $x_{1}, \ldots, x_{n}$ is the complete list of the variables that occur in $P$, then. $P\left(t_{1}, \ldots, t_{n}\right)$ is the result of substituting variables $x_{i}$ by terms $t_{i}$ in $P$. We can then formulate the problem of pattern matching as follows: 
Let $\underline{\text { pat }}=\operatorname{pat}\left(x_{1}, \ldots, x_{n}\right)$

Find $z$ such that

$$
\begin{aligned}
\text { if } \underline{\arg }=\operatorname{pat}\left(t_{1}, \ldots, t_{n}\right) \text { for some } t_{1}, \ldots, t_{n} \\
\text { then } z=\left\{\left\langle x_{1} t_{1}>, \ldots,<x_{n} t_{n}\right\rangle\right\} \\
\text { else } x=\text { NOMATCH. }
\end{aligned}
$$

Note that this specification includes implicityly the restriction that the match found be a most general match, because each of the variables $x_{i}$ actually occurs in pat. Therefore, the specifications do not need to be strengthened during the course of the synthesis.

We hope to experiment with visual representations in a variaty of applications. Clearly, while the reasoning required is simplified by the use of pictorial notation, the handing of innovations such as the ellipsis notation in an implementation is conrespondingly more complex.

\section{ACKNOWLEDGEMENTS}

We wish to thank Robert Boyer, Bertram Raphael, and Georgia Sutherland for giving detailed critical readings of the manuscript. We would also like to thank Nachum Dershowitz, Peter Deutsch, Richard Fikes, Akira Fusaoka, Cordell Green and his students, Irene Greif, Carl Hewitt, Shmuel Katz, David Luckham, Earl Sacerdoti, and Ben Wegbreit for conversations that aided in formulating the ideas in this paper. We would also like to thank Claire Collins and Hanna Zies for typing many versions of this manuscript.

This research was primarily sponsored by the National Science Foundation under grants GJ-36I46 and GK-35493. 


\section{BIBLIOGRAPHY}

1. Balzer, R. M. (September 1972), "Automatic Programming," Institute Technical Memo, Univensity of Southern California/Information Sciences Institute.

2. Biermann, A. W., R. Baum, R. Kirisknaswamy and F. E. Petry coctober 1973), "Automatic Program Synthesis Reports," Computer and Information Sciences Technical Report TR-73-6, Ohio State University.

3. Bobrow, D. G. and B. Wegbreit (August 1973), "A Model fon Control Structures for Artificial Intelligence Programming Languages," Adv. Papers 3d. Intl. Conf. on Artificial Intelligence, 246253, Stanford University, Stanford, California.

4. Boyer, R. S. and J S. Moore (1973), "Proving Theorems about LISP Functions," Adv. Papers 3d. Intl. Conf. on Artificial Intelizigence.

5. Buchanan, J. R. and D. C. Luckham (March 1974), "On Automating the Construction of Programs," Memo, Stanford Artificial Intelligence Project, Stanford, California.

6. Bundy, A. (August 1973), "Doing Arithmetic with Diagrams," Adv. Papers 3d. Intl. Conf. on Artificial Intelligence, 130-138, Stanford University, Stanford, California.

7. Floyd, R. W., (1967), "Assigning Meanings to Programs," Proc. of a Symposium in Applied Mathematics, Vol. 19, (J. T. Schwantz, ed.), Am. Math. Soc., 19-32.

8. Green, C. C. (May 1969), "Application of Theorem Proving to Problem Solving," Proc. Intl. Joint Conf. on Artificial Inteliigence, 219-239.

9. Green, C. C., R. Waldinger, R. Elschlager, D. Lenat, B. Mccune, and D. Shaw, (1974), "Progress Report on Program-Understanding Programs," Memo, Stanford Artificial Intelligence Project, Stanford, California.

10. Hardy, S. (December 1973), "Automatic Induction of LISP Functions," Essex University. 
1. Hewitt, C. (1972), "Description and Theoretical Analysis (Using Schemata) of PLANNER: A Language for Proving Theorems and Manipulating Models in a Robot," AI Memo No. 251, MIT, Project MAC, April 1972.

12. Hoare, C. A. R., (October 1969), "An Axiomatic Basis for Computer Programming," C. ACM 12, 10, 576-580, 583 .

13. Kowalski, R. (March 1974), "Logic for Problem Solving," Memo No. 75, Department of Computational Logic, University of Edinburgh, Edinburgh.

14. Luckham, D. and J. R. Buchanan (March 1974), "Automatic Generation of Programs Containing Conditional Statements," Memo, Stanford Artificial Intelligence Project, Stanford, California.

15. Manna, Z. and R. Waldinger (March 1971), "Toward Automatic Program Synthesis," Comm. ACM, Vol. 14, No. 3, PP. 151-165.

16. McCarthy, J. (1962), "Towards a Mathematical Science of Computation," Proc. IFIP Congress 62, North Holland, Amsterdam, 21-28.

17. Reboh, R. and E. Sacerdoti (August 1973), "A Preliminary QIISP Manual, "Tech. Note 81, Artificial Intelligence Center, Stanford Research Institute, Menlo Park, California.

18. Robinson, J. A., (January 1965), "A Machine-Oriented Logic Based on the Resolution Principle," Jour. ACM, Vol. 12, No. 1, 23-41.

19. Rulifson, J. F., J.A. Derksen, and R. J. Waldinger (November 1972), "QA4: A Procedural Calculus for Intuitive Reasoning," Tech. Note 73, Artificial Intelligence Group, Stanford Research Institute, Menlo Park, California.

20. Simon, H. A., (October 1963), "Experiments with a Heuristic Comuter," Jour. ACM, Vol. 10, No. 4, 493-506.

21. Sussman, G. J. (August 1973), "A Computational Model of Skill Acquisition," Ph.D. Thesis, Artificial Intelligence Laboratory, M.I.T., Cambridge, Mass.

22. Teitelman, W., (1974), INTERLISP Reference Manual, Xerox, PaIo Alto, California.

23. Waldinger, R. J., and R. C. T. Lee (May 1969), "PRoW: A Step Toward Automatic Program Writing," Proc. Int1. Joint Conf. on Artificial Intelligence, 241-252. 